\title{
Plasma Hsa-miR-92a-3p in correlation with lipocalin-2 is associated with sepsis-induced coagulopathy
}

Yishan Wang ${ }^{1 \dagger}$, Huijuan Wang ${ }^{1 \dagger}$, Chunfang Zhang ${ }^{2}$, Chao Zhang ${ }^{1}$, Huqin Yang ${ }^{1}$, Ruiyue Gao ${ }^{1}$ and Zhaohui Tong ${ }^{1 *}$

\begin{abstract}
Background: Sepsis is a life-threatening situation, and it can be rendered more severe by coagulopathy. We here examine a novel plasma biomarker for sepsis-induced coagulopathy.

Methods: A total of 116 patients diagnosed with sepsis were recruited and divided into two groups by whether they also had coagulopathy. Plasma samples were collected on arrival at the intensive care unit. Fifteen sepsis-alone and 15 sepsis-induced coagulopathy plasma samples were mixed and sent for microRNA sequencing. Differently expressed microRNAs were then validated by quantitative reverse transcriptase polymerase chain reaction in 52 sepsis-alone and 34 sepsis-induced coagulopathy patients; plasma lipocalin-2 was measured as well.

Results: Four microRNAs were selected from microRNA sequencing. Only hsa-mir-92a-3p was differently expressed in the validation set. Its level of expression was significantly lower in sepsis-induced coagulopathy group. Hsa-mir92a-3p had an area under a receiver operating characteristic curve of 0.660 (95\% confidence interval, $0.537,0.782$ ). The plasma Hsa-mir-92a-3p level was related to activated partial thromboplastin time, prothrombin activity, and plasma lipocalin-2 level. A binary logistic model showed an association between hsa-mir-92a-3p and fibrinogen with SIC.

Conclusions: The utility of hsa-mir-92a-3p as a biomarker for sepsis-induced coagulopathy needs more verification, and the regulatory mechanism of hsa-mir-92a-3p in coagulation disorder and its potency as a therapeutic target must be confirmed.
\end{abstract}

Keywords: Sepsis, Coagulation disorder, microRNA, Biomarker, Diagnosis

\section{Background}

Sepsis is a series of life-threatening organ dysfunction events initiated by dysregulated host response to infection [1]. Disseminated intravascular coagulation (DIC) is a major cause of organ dysfunction in sepsis. It is significantly closely related to mortality and difficult to be recognized before coagulation collapsing [2, 3]. Recently, multiple studies have confirmed that early diagnosis and intervention of DIC is crucial to disease prognosis [4-9],

\footnotetext{
* Correspondence: tongzhaohuicy@sina.com

${ }^{\dagger}$ Yishan Wang and Huijuan Wang contributed equally to this work.

${ }^{1}$ Department of Respiratory and Critical Care Medicine, Beijing Engineering Research Center of Respiratory and Critical Care Medicine, Beijing Chao-Yang Hospital, Capital Medical University, Beijing Institute of Respiratory Medicine, NO. 8, Gong Ti South Road, Chao-Yang District, Beijing 100020, China Full list of author information is available at the end of the article
}

which highlighted the importance of identifying sepsisinduced coagulopathy (SIC) before it is too late.

MicroRNA (miRNA) is a group of endogenous noncoding small RNAs. They are highly conserved and function as posttranscriptional regulators of gene expression [10]. It has been reported that levels of miRNAs are stable across the human circulatory system [11, 12]. MiRNAs have been studied widely as biomarkers for several diseases. MiR-146a, miR-150, miR-4772-5p, miR15a, miR-223, and miR-499-5p are potential biomarkers for sepsis in human serum/plasma, and down-regulation of miR-574-5p has been shown to be associated with higher risk of mortality [13-18]. However, there has been little study of miRNAs for SIC, according to Wang et al. Some miRNAs have been found to be related to sepsis prognosis. Among these, serum miR-122 was 
found to be related to coagulation disorder in sepsis patients. Thus, studies on miRNAs in association with SIC are sorely needed.

Lipocalin-2, also called neutrophil gelatinaseassociated lipocalin (NGAL), is a $25 \mathrm{kD}$ protein secreted by human neutrophils. Lipocalin-2 is widely used as an index in clinical settings. It can be detected in serum and plasma samples easily and quickly in many inflammatory and metabolic disorders. Its relationship with sepsis has been studied extensively. It has been used as a biomarker for sepsis-associated acute kidney injury and severe sepsis prognosis [19]. One recent study revealed its regulatory role in gut-origin sepsis [20]. Another study indicated that plasma lipocalin-2 levels are elevated in DIC patients and related to disease prognosis [21]. Though the relationship of lipocalin-2 with sepsis and DIC has been studied, no study has yet evaluated its relationship with SIC.

In this study, we sequenced plasma microRNA in sepsis-alone and SIC patients. Four microRNAs were found to be differently expressed including hsa-mir-92a$3 p$. In the subsequent PCR validation, we observed the hsa-mir-92a-3p expression level was consistent with sequencing results and was associated with plasma lipocalin-2 levels. We then explored the potential of hsamir-92a and lipocalin-2 as disease-specific markers.

\section{Methods}

\section{Study design and patient characteristics}

Patients were diagnosed with sepsis according to "The Third International Consensus Definitions for Sepsis and Septic Shock (Sepsis-3)" [1]. Exclusion criteria were as follows: (1) younger than 18 years old, (2) immunocompromised status, (3) pregnancy, (4) malignancy, (5) massive transfusion within $24 \mathrm{~h}$ of sample collection.

A total of 116 patients (49 SIC and 67 sepsis-alone) were divided into two groups: SIC group and sepsisalone (SA) group. SIC was defined as proposed by Iba et al. in 2017 [22]: SIC scores no less than 4 points with total score of prothrombin time and coagulation exceeding 2 points. The SIC score system (Table 1) consisted of three parts: Prothrombin time, which was assessed using PT-INR value; coagulation, assessed by platelet count; and total SOFA, calculated using 4 items (respiratory SOFA, cardiovascular SOFA, hepatic SOFA, and

Table 1 Score system for sepsis-induced coagulopathy

\begin{tabular}{lllll}
\hline Category & Parameter & 0 & 1 & 2 \\
\hline Prothrombin time & PT-INR & $\leq 1.2$ & $>1.2$ & $>1.4$ \\
Coagulation & Platelet count $\left(\times 10^{9} / \mathrm{L}\right)$ & $\geq 150$ & $<150$ & $<100$ \\
Total SOFA & SOFA four items & 0 & 1 & $\geq 2$ \\
\hline
\end{tabular}

SOFA Sequencing Organ Failure Assessment renal SOFA). The SIC score system was proved to be closely correlated with mortality rate. It took the SOFA score into consideration, which was tailored for sepsisassociated coagulopathy [22-24].

\section{Blood sample and plasma MiRNA isolation}

Blood samples were collected from all patients within $24 \mathrm{~h}$ of admission to the intensive care unit (ICU) and centrifuged at $3000 \mathrm{rpm}$ for $15 \mathrm{~min}$ at room temperature. The supernatant was transferred to Eppendorf tubes and stored at $-80^{\circ} \mathrm{C}$ until miRNA extraction. Total plasma miRNA was isolated using a serum/plasma miRNA isolation and extraction kit (Tiangen, Beijing, China) according to the manufacturer's instructions.

\section{Hiseq sequencing and target MiRNA selection}

The plasma samples of 15 SIC patients and 15 SA patients were pooled in two separate groups. Total miRNA were isolated using miRcute miRNA extraction and isolation kit (Tiangen, Beijing, China); gene sequencing was completed by the Beijing Genomics Institute (BGI, Beijing, China).

MiRNAs selected for further validating all met the following two criteria (1) over 20 read numbers; (2) $\log 2 \mathrm{Ra}$ tio $\geq 2[25,26]$.

\section{Target MiRNA validation via qRT-PCR}

CDNA was synthesized using a miRcute Enhanced miRNA cDNA First-line Synthesis Kit (kr211) and realtime qPCR was performed with a SYBR Green miRNA assay (Tiangen, Beijing, China). Briefly, $8 \mu \mathrm{l}$ of isolated miRNA was added to a $20 \mu \mathrm{l}$ system, $42^{\circ} \mathrm{C}$ for $60 \mathrm{~min}$ followed by $95^{\circ} \mathrm{C} 3 \mathrm{~min}$ for reverse transcription. Two $\mu \mathrm{l}$ of cDNA was added to a 20- $\mu$ l system tested with ABI 7500 Real-time PCR System in duplication. The program was set according to the manufacture's instruction.

\section{Plasma Lipocalin-2 assessment}

Lipocalin-2 levels were measured using an enzyme-linked immunosorbent assay (ELISA) kit (Life Technologies, CA, US) with a range of 7.81 to $500 \mathrm{pg} / \mathrm{ml}$. Concentration was determined from standard curves. ELISA was performed in duplicate according to the manufacturer's instructions.

\section{Statistical analysis}

Expression levels of selected miRNAs detected by qRT-PCR are here presented with raw $\mathrm{Ct}$ values. Results for normally distributed continuous variables were given as means \pm standard errors and compared between groups using Student's $t$ tests. Results for non-normally distributed continuous variables are summarized as medians (interquartile ranges) and compared using the Mann-Whitney U tests. Pearson's analysis was used to evaluate the relationships 
Table 2 Patients' clinical characteristics for validation set $(n=$ 86)

\begin{tabular}{|c|c|c|c|}
\hline Variables & $\begin{array}{l}\text { Sepsis-induced } \\
\text { coagulopathy }(n=34)\end{array}$ & $\begin{array}{l}\text { Sepsis alone } \\
(n=52)\end{array}$ & $P$-value \\
\hline Sex, F/M & $61.90 \%$ & $62.50 \%$ & $P=0.983^{a}$ \\
\hline Age, years & $59.56 \pm 17.131$ & $63.48 \pm 17.927$ & $P=0.316^{b}$ \\
\hline \multicolumn{4}{|c|}{ Source of infection\% } \\
\hline Pulmonary & $58.00 \%(29)$ & $42.00 \%(21)$ & $P<0.001^{a}$ \\
\hline $\begin{array}{l}\text { Biliary } \\
\text { system and } \\
\text { pancreatic }\end{array}$ & $45.50 \%(5)$ & $54.50 \%(6)$ & $P=0.276^{a}$ \\
\hline Peritonitis & $78.90 \%(15)$ & $21.10 \%(4)$ & $P<0.001^{a}$ \\
\hline $\begin{array}{l}\text { Urinary } \\
\text { system }\end{array}$ & $60.00 \%(3)$ & $40.00 \%(2)$ & $P=0.380^{a}$ \\
\hline Cerebral & $0 \%(0)$ & $100 \%(1)$ & $P=1.000^{\mathrm{a}}$ \\
\hline SOFA scores & $6.68 \pm 3.67$ & $6.04 \pm 3.16$ & $P=0.393^{b}$ \\
\hline $\begin{array}{l}\text { APACHE II } \\
\text { scores }\end{array}$ & $17.35 \pm 7.88$ & $16.60 \pm 6.73$ & $P=0.635^{b}$ \\
\hline $\begin{array}{l}\text { 28-day } \\
\text { mortality\% }\end{array}$ & $67.7 \%(21)$ & $32.3 \%(10)$ & $P<0.001^{a}$ \\
\hline PLT (×10^9/L) & $108.03 \pm 91.99$ & $211.10 \pm 96.93$ & $P<0.001^{b}$ \\
\hline $\begin{array}{l}\text { D-dimer (mg/ } \\
\mathrm{dL} \text { ) }\end{array}$ & $7.12(3.00,18.24)$ & $4.60(2.38,8.64)$ & $P<0.001^{c}$ \\
\hline APTT (s) & $44.80(38.10,61.10)$ & $35.75(31.70,43.23)$ & $P<.001^{c}$ \\
\hline PT (s) & $14.90(13.48,17.33)$ & $12.75(11.53,13.88)$ & $P<0.001^{c}$ \\
\hline PA\% & $66.53 \pm 17.89$ & $81.10 \pm 11.98$ & $P<0.001^{c}$ \\
\hline INR & $1.26(1.16,1.47)$ & $1.11(1.03,1.18)$ & $P<0.001^{c}$ \\
\hline $\mathrm{Fbg}(\mathrm{mg} / \mathrm{dL})$ & $275.13 \pm 136.45$ & $421.50 \pm 173.30$ & $P<0.001^{b}$ \\
\hline$\Pi(\mathrm{s})$ & $20.00(18.28,24.88)$ & $19.10(17.98,20.70)$ & $P=0.055^{c}$ \\
\hline $\mathrm{Cr}(\mathrm{mmol} / \mathrm{L})$ & $75.65(52.90,137.20)$ & $74.25(53.80,127.65)$ & $P=0.575^{c}$ \\
\hline BUN (mmol/L) & $10.12(5.78,14.07)$ & $8.54(5.06,12.78)$ & $P=0.299^{c}$ \\
\hline
\end{tabular}

${ }^{\mathrm{a}} \mathrm{X} 2$ test, ${ }^{\mathrm{b}}$ Student's $\mathrm{t}$ test, ${ }^{\mathrm{c}}$ Mann-Whitney $\mathrm{U}$ test, SOFA Sequential organ failure assessment, APACHE II Acute physiology and chronic health evaluation, PLT Platelet, APTT activated partial thromboplastin time, PT Prothrombin time, PA Prothrombin activity, INR International normalized ratio, Fbg Fibrinogen, $\Pi T$ Thrombin Time, $\mathrm{Cr}$ Creatine, BUN Blood urea nitrogen

between pairs of variables. Statistical significance was set at $P<0.05$. SPSS 20.0 software was used for all statistical analyses.

A binary logistic regression model was generated after the following hypothesis was tested: (1) There is a linear relationship between the continuous independent variable and the logit conversion value of the dependent variable; (2) There is no multicollinearity between independent variables; (3) There are no obvious outliers, leverage points, or strong influence points. The variables that showed statistically significant differences in univariate analysis were entered into the binary logistic regression model. Variables were excluded for existence of multicollinearity between independent variables. Finally, six variables were taken into the final analysis as shown in the results.

\section{Results}

Patients

A total of 116 sepsis patients were included between Nov 2014 and Aug 2017 from emergency intensive care units, respiratory intensive care units, and surgery intensive care units of Beijing Chao-Yang Hospital. Eighty-six patients were included for the validation set and were divided into SIC group $(n=34)$ and SA group $(n=52)$. Patients' clinical characteristics are presented in Table 2 . Patients from the two groups were matched by sex $(P=0.983)$, age $(P=$ $0.316)$ and disease severity measured by Acute Physiology and Chronic Health Evaluation (APACHE) II scores $(P=$ 0.635). Sources of infection causing sepsis included pulmonary infection, biliary and pancreatic infection, peritonitis, urinary infection, and cerebral infection. There were significantly more cases of pulmonary infection $(P<0.001)$

Table 3 Patients' clinical characteristics for gene sequencing $(n=30)$

\begin{tabular}{|c|c|c|c|}
\hline Variables & $\begin{array}{l}\text { Sepsis-induced } \\
\text { coagulopathy ( } n=15 \text { ) }\end{array}$ & $\begin{array}{l}\text { Sepsis alone } \\
(n=15)\end{array}$ & $\begin{array}{l}P \text { - } \\
\text { value }\end{array}$ \\
\hline Sex, F/M & $4 / 11$ & $8 / 7$ & $P=0.136^{\mathrm{a}}$ \\
\hline Age, years & $62.60 \pm 13.91$ & $65.13 \pm 20.40$ & $P=0.694^{b}$ \\
\hline \multicolumn{4}{|c|}{ Cause of sepsis\% } \\
\hline $\begin{array}{l}\text { Pulmonary } \\
\text { infection }\end{array}$ & $75 \%(9)$ & $25 \%(3)$ & $P=0.025^{a}$ \\
\hline $\begin{array}{l}\text { Biliary } \\
\text { system and } \\
\text { pancreatic }\end{array}$ & $20 \%(1)$ & $80 \%(4)$ & $P=0.330^{\mathrm{a}}$ \\
\hline Peritonitis & $40 \%(4)$ & $60 \%(6)$ & $P=0.439^{a}$ \\
\hline $\begin{array}{l}\text { Urinary } \\
\text { system }\end{array}$ & $50 \%(1)$ & $50 \%(1)$ & $P=1.000^{\mathrm{a}}$ \\
\hline Cerebral & $0 \%(0)$ & $100 \%(1)$ & $P=1.000^{\mathrm{a}}$ \\
\hline SOFA scores & $9.00 \pm 4.33$ & $6.21 \pm 3.39$ & $P=0.060^{b}$ \\
\hline $\begin{array}{l}\text { APACHE ॥ } \\
\text { scores }\end{array}$ & $20.20 \pm 8.19$ & $15.59 \pm 7.77$ & $P=0.125^{b}$ \\
\hline $\begin{array}{l}\text { PLT }(\times 10 \wedge 9 / \\
\text { L) }\end{array}$ & $131.20 \pm 87.78$ & $156.87 \pm 76.27$ & $P=0.400^{b}$ \\
\hline $\begin{array}{l}\text { D-dimer (mg/ } \\
\mathrm{dL} \text { ) }\end{array}$ & $13.94 \pm 12.41$ & $5.72 \pm 5.49$ & $P=0.026^{b}$ \\
\hline APTT (s) & $50.80(39.70,60.70)$ & $34.90(33.40,53.20)$ & $P=0.062^{c}$ \\
\hline PT (s) & $17.00(14.20,20.10)$ & $12.90(12.50,18.00)$ & $P=0.022^{c}$ \\
\hline PA\% & $62.15 \pm 19.45$ & $70.51 \pm 14.57$ & $P=0.193^{b}$ \\
\hline INR & $1.51 \pm 0.54$ & $1.30 \pm 0.29$ & $P=0.197^{b}$ \\
\hline $\mathrm{Fbg}(\mathrm{mg} / \mathrm{dL})$ & $294.60(140.60,340.80)$ & $310.30(254.50,533.40)$ & $P=0.262^{c}$ \\
\hline$\Pi(\mathrm{s})$ & $21.50(19.20,114.45)$ & $23.00(18.70,282.90)$ & $P=0.787^{c}$ \\
\hline $\mathrm{Cr}(\mathrm{mmol} / \mathrm{L})$ & $137.40(74.05,232.75)$ & $129.56(77.80,301.15)$ & $P=0.967^{c}$ \\
\hline $\begin{array}{l}\text { BUN (mmol/ } \\
\text { L) }\end{array}$ & $11.32 \pm 6.49$ & $11.45 \pm 6.38$ & $P=0.959^{b}$ \\
\hline
\end{tabular}

${ }^{\mathrm{a}} \mathrm{X} 2$ test, ${ }^{\mathrm{b}}$ Student's $\mathrm{t}$ test, ${ }^{\mathrm{C}}$ Mann-Whitney $\mathrm{U}$ test, SOFA Sequential organ failure assessment, APACHE /I Acute physiology and chronic health evaluation, PLT Platelet, APTT Activated partial thromboplastin time, PT Prothrombin time, $P A$ Prothrombin activity, INR International normalized ratio, Fbg Fibrinogen, $\Pi T$ Thrombin Time, $\mathrm{Cr}$ Creatine, BUN Blood urea nitrogen 
Table 4 Differently expressed miRNAs in miRNAs sequencing

\begin{tabular}{llllll}
\hline miR_name & SA Reads & SIC Reads & log2Ratio & $P$-value & q-value \\
\hline hsa-miR-92a-3p & 9184 & 9651 & -4.96 & 0 & 0 \\
hsa-miR-143-3p & 5959 & 9641 & -4.33 & 0 & 0 \\
hsa-miR-30c-5p & 645 & 335 & -5.97 & 0 & 0 \\
hsa-miR-185-5p & 663 & 2718 & -2.99 & 0 & 0 \\
\hline
\end{tabular}

miR microRNA, SA Sepsis Along, SIC Sepsis Induced Coagulopathy and peritonitis $(P<0.001)$ in the SIC group than in the SA group. Patients were matched for other causes: biliary system and pancreatic infection $(P=0.276)$, urinary infection $(P=0.380)$, and cerebral infection $(P=1.000)$. Most global coagulation tests differed significantly between the two groups except $\mathrm{D}$-dimer $(P=0.097)$ and thrombin time $(\mathrm{TT})$ $(P=0.101)$. There was no difference between the two groups with respect to creatinine $(P=0.575)$ or blood urea nitrogen $(\mathrm{BUN})(P=0.299)$ levels.

Another 30 patients (15 SIC patients and 15 SA patients) were included for gene sequencing, demographic variables were presented in Table 3.

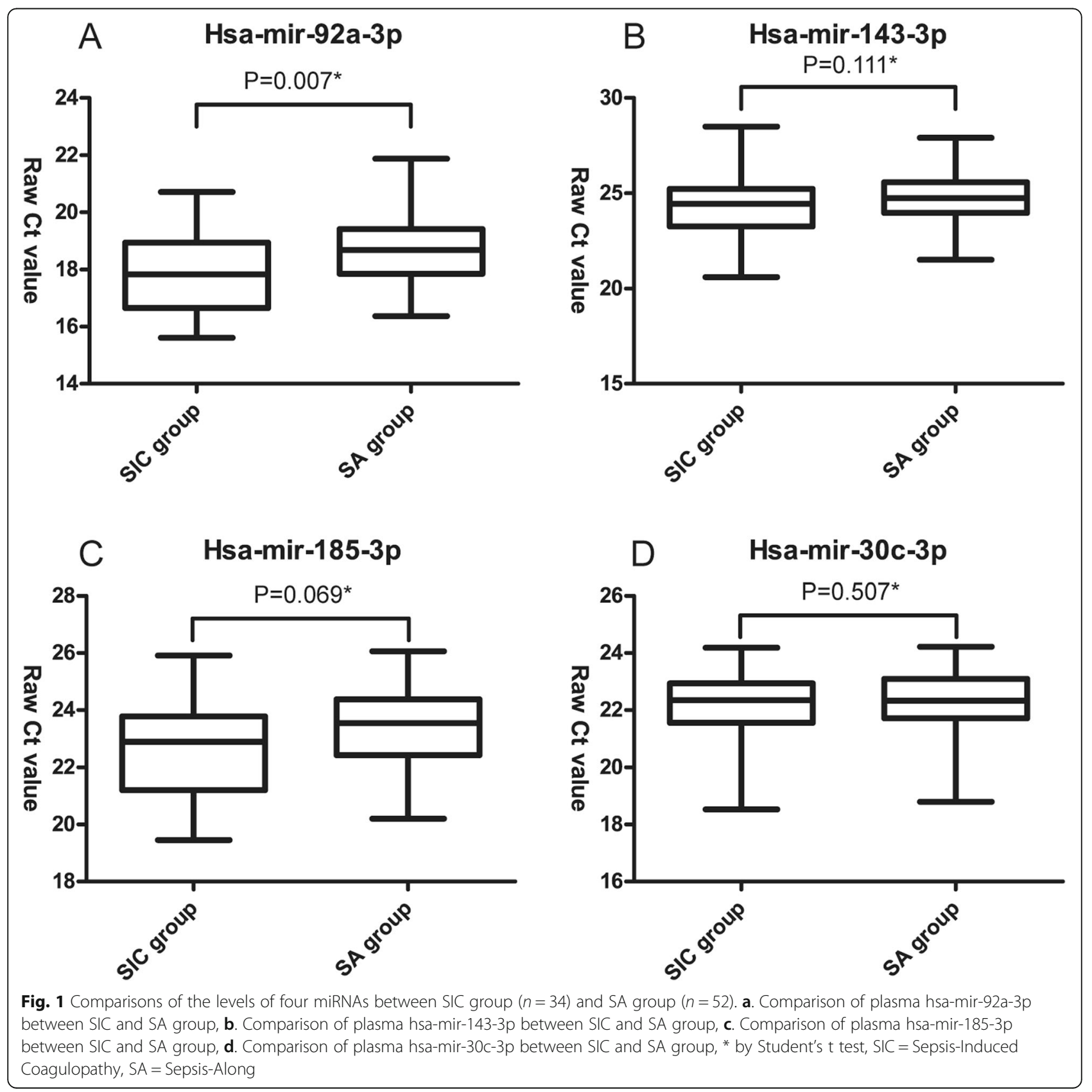




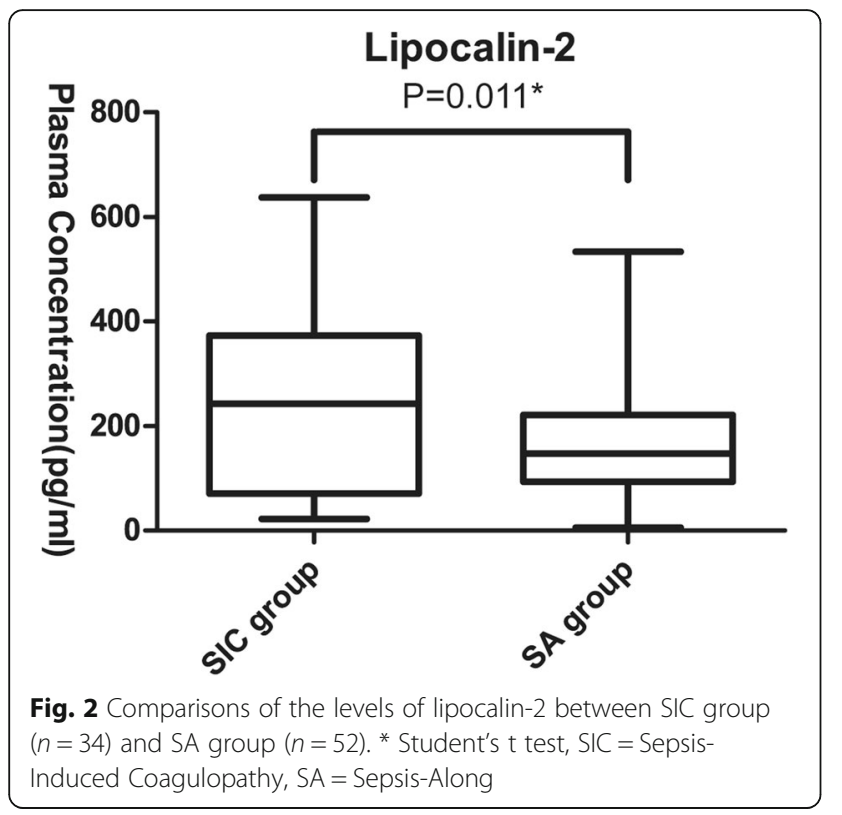

Hsa-mir-92a-3p expressed differently between SIC group and SA group

According to the results of gene sequencing, we chose hsa-miR-143-3p, hsa-miR-185-3p, hsa-miR-92a-3p, and hsa-miR-30c-3p for further measurement; mean reads and $\log 2$ ratio of four miRNAs are shown in Table 4 . The Student's $t$ test revealed significance only for level of hsa-mir-92a-3p: SIC group vs. SA group $(17.86 \pm 1.44$ vs. $18.66 \pm 1.21, P=0.007$ ) (Fig. 1).

\section{Plasma Hsa-mir-92a level related to global coagulation index}

We recorded patients' global coagulation tests results including D-dimer, activated partial thromboplastin time (APTT), prothrombin activity (PA), fibrinogen (FBG), and thrombin time (TT). Statistical analysis revealed a strong relationship between hsa-mir-92a and PA (Pearson's correlation coefficients $=0.266, P=0.013$ ) and hsa-mir-92a and APTT (Pearson's correlation coefficients $=-0.325, P=0.002$ ).

\section{Plasma Hsa-miR-92a level related to plasma Lipocalin-2 level}

We measured plasma lipocalin-2 levels and found plasma lipocalin-2 expression to differ between the SIC and SA groups $(246.05 \pm 169.95 \mathrm{pg} / \mathrm{ml}$ vs. $168.84 \pm$ $105.30 \mathrm{pg} / \mathrm{ml}, P=0.011$ ) (Fig. 2) and to be related to plasma hsa-miR-92a level (Pearson's correlation coefficients $=-0.282, P=0.009$ ).

\section{Diagnostic values of Hsa-miR-92a and Lipocalin-2}

Results showed hsa-miR-92a and lipocalin-2 could be used to distinguish sepsis-induced coagulopathy from sepsis-alone patients. To compare the diagnostic values of these two biomarkers, receiver operating characteristic (ROC) curves were generated and area under the curve (AUC) was calculated. Hsa-miR-92a had an AUC of $0.660(P=0.011,95 \% \mathrm{CI}$,

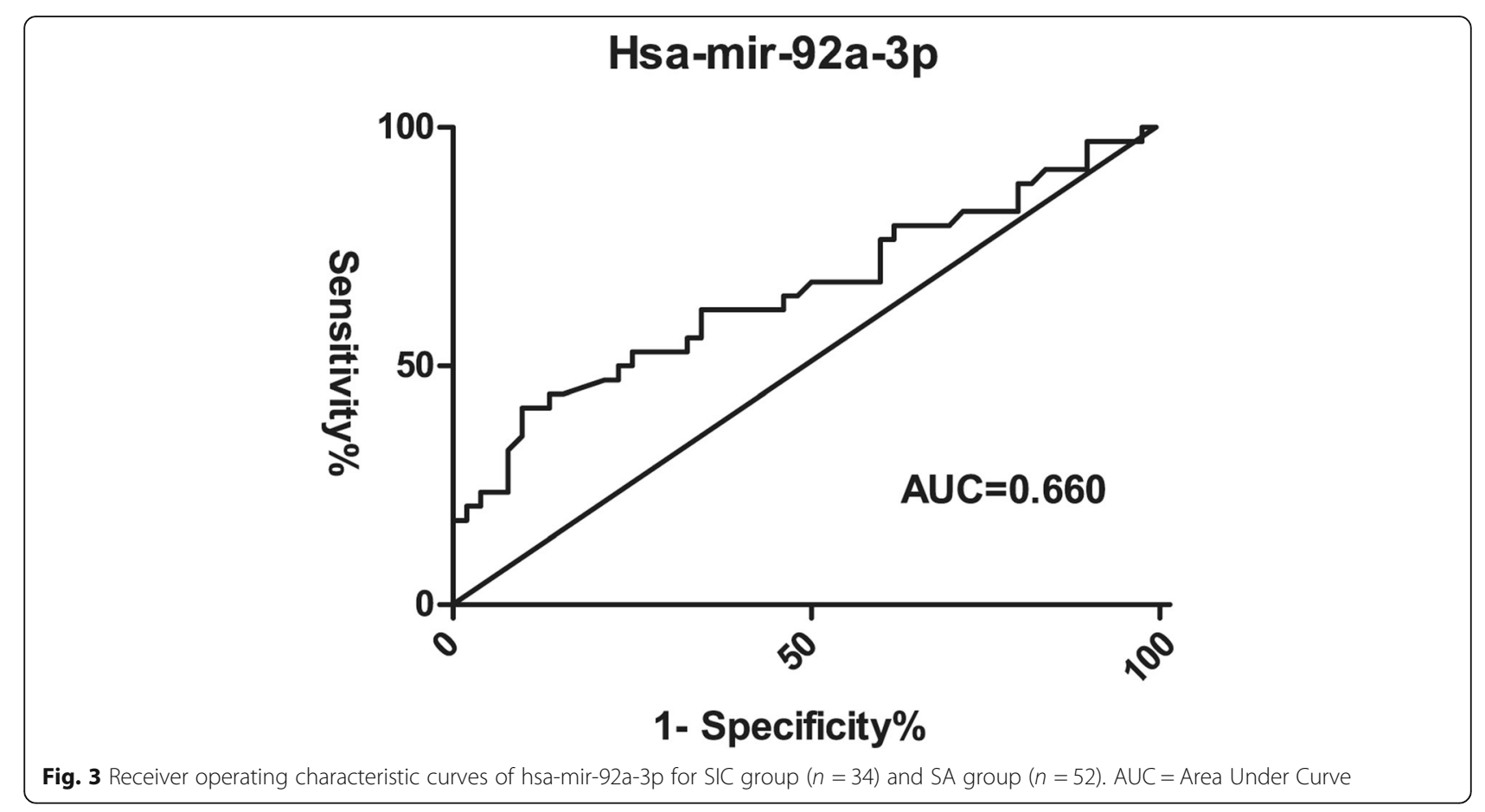




\section{Lipocalin-2}

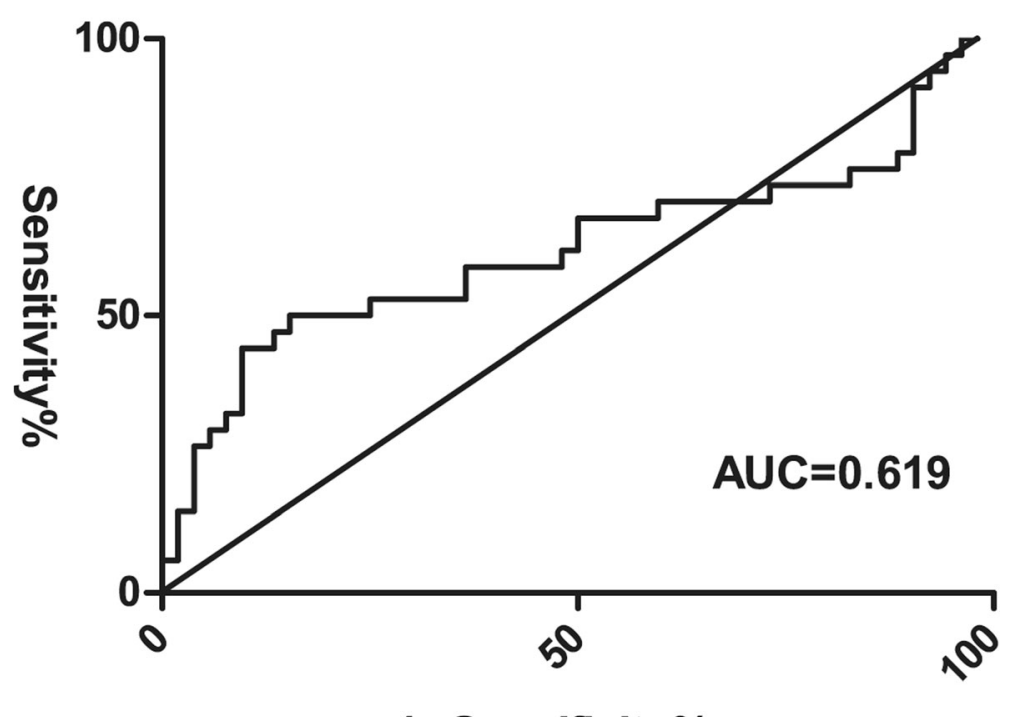

1- Specificity\%

Fig. 4 Receiver operating characteristic curves of lipocalin-2 for SIC group $(n=34)$ and SA group $(n=52)$. AUC = Area Under Curve

$0.537,0.782)$ in predicting the absence of SIC whereas lipocalin-2 had an AUC of 0.619 ( $P=0.084,95 \%$ CI, 0.485, 0.752 ) in predicting SIC (Fig. 3, Fig. 4). When the cutoff point was set at $261.01 \mathrm{pg} / \mathrm{mL}$, lipocalin-2 had the highest specificity of $84.6 \%$ (Table 5 ).

We then generated the ROC for a combination of hsamir-92a and lipocalin-2 (Fig. 5). The AUC for hsa-mir$92 \mathrm{a}+$ lipocalin-2 was $0.687(P=0.003,95 \% \mathrm{CI}, 0.578$, $0.782)$.

\section{Risk factors for Sepsis-induced coagulopathy}

As shown, hsa-mir-92a was differently expressed between SIC and SA groups. Because we hypothesized sepsis accompanied by coagulation disorder indicated more disease severity, APACHEII score was also taken into analysis. The analysis of basic information indicated a difference in the number of patients with each specific source of pulmonary infection and peritonitis; these variables were taken into a binary logistic regression model.

Finally, we used a binary logistic regression model to assess the influence of the plasma level of hsa-mir-92a, APACHEII score, pulmonary infection and peritonitis, D-dimer level, and fibrinogen for SIC. There were linear relationships between dependent variables and covariates and one case with a ZResid of 3.386 times the standard deviation. These were analyzed further. The model was significant, 0.531 for Hosmer and Lemeshow goodness of fit test. Among the six independent variables included in the model, mir-92a and fibrinogen had statistical significance. For each unit of increase in plasma mir-92a level, the risk of SIC decreased by $32.4 \%$, and for each unit of increase in plasma fibrinogen level, the risk of SIC decreased by $0.7 \%$. The result and odds ratio with its $95 \% \mathrm{CI}$ are given in Table 6.

\section{Discussion}

Sepsis is a life-threatening situation. It is often accompanied by coagulation disorder, and it can leave clinicians stymied in the attempt to identify the optimum treatment. It would be useful to find new biomarkers and therapeutic targets. In this study, we found novel biomarkers for SIC. After Hiseq sequencing, we located four differently expressed miRNAs in human plasma and validated our findings in a larger sample. Plasma lipocalin-2 was measured as well. We found significant differences in both hsa-mir-92a and

Table 5 Diagnostic values of has-miR-92a and lipocalin-2

\begin{tabular}{lllll}
\hline Biomarker & AUC $(95 \% \mathrm{Cl})$ & Cutoff point & Sensitivity\% & Specificity\% \\
\hline Hsa-miR-92a & $0.660(0.537,0.782)$ & 17.18 & $41.20 \%$ & $58.80 \%$ \\
lipocalin-2 & $0.619(0.485,0.752)$ & $261.01 \mathrm{pg} / \mathrm{mL}$ & $50 \%$ & $84.60 \%$ \\
Hsa-miR-92a + lipocalin-2 & $0.687(0.578,0.782)$ & 0.46139 & $52.94 \%$ & $84.62 \%$ \\
\hline
\end{tabular}




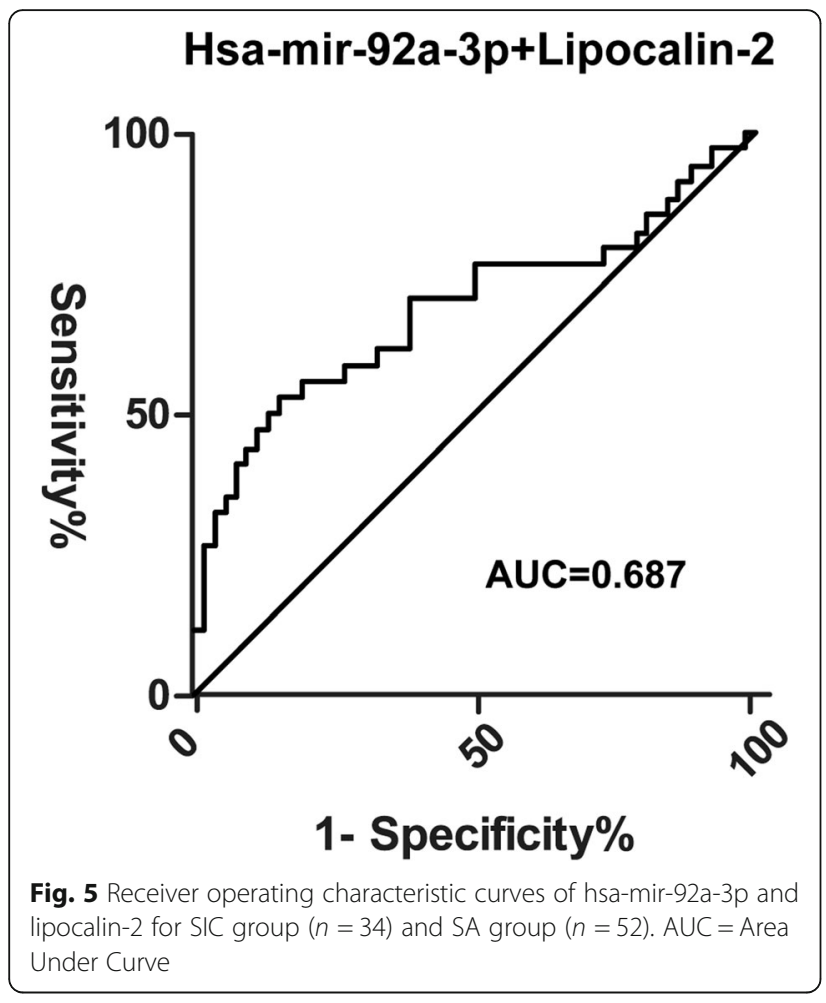

lipocalin-2 levels between SIC and SA groups. There were higher plasma levels of hsa-mir-92a in the SA group than in the SIC group, but the higher plasma lipocalin-2 level indicated a greater possibility of SIC. We also found a strong relationship between hsa-mir-92a and prothrombin activity, activated partial thromboplastin time, and lipocalin-2.

MiR-92a has been widely studied in tumorigenesis and metastasis of several different kinds of tumors. MiR-92a is significantly up-regulated in colon rectal cancer tissues and correlated with metastases and poor prognosis [27]. It also participates in gene regulation processes in other kinds of tumors including non-Hodgkin's lymphoma,

Table 6 Risk factors for sepsis-induced coagulopathy

\begin{tabular}{|c|c|c|c|c|c|}
\hline \multirow[b]{2}{*}{ Variables } & \multirow[b]{2}{*}{ DF } & \multirow[b]{2}{*}{$P$ value } & \multirow[b]{2}{*}{ OR } & \multicolumn{2}{|l|}{$95 \% \mathrm{Cl}$} \\
\hline & & & & Lower & Upper \\
\hline Hsa-mir-92a & 1 & 0.035 & 0.658 & 0.446 & 0.970 \\
\hline Fibrinogen & 1 & 0.001 & 0.993 & 0.989 & 0.997 \\
\hline D-dimer & 1 & 0.093 & 1.050 & 0.992 & 1.111 \\
\hline APACHEII & 1 & 0.937 & 1.003 & 0.932 & 1.080 \\
\hline Pulmonary Infection & 1 & 0.402 & 0.600 & 0.182 & 1.979 \\
\hline Peritonitis & 1 & 0.857 & 0.853 & 0.150 & 4.856 \\
\hline Constant & 1 & 0.019 & $10,504.227$ & & \\
\hline
\end{tabular}

The binary logistic model included six variables: plasma level of hsa-mir-92a, Fibrinogen, D-dimer, APACHE II score, pulmonary infection and Peritonitis. APACHEIl Acute physiology and chronic health evaluation, DF Degree of freedom, OR Odds ratio, Cl Confident interval acute lymphoid leukemia, multiple myeloma, breast cancer, lung cancers, hepatocellular carcinoma, and esophageal squamous cell carcinoma, acting as both tumor suppressor and oncogene [28-34].

Mir-92a was found to be involved in angiogenesis and endothelial cell function [35]. Bonauer et al. found that human endothelial cells (ECs) express the miR-17-92 cluster, particularly its member miR-92a. Forced overexpression of miR-92a in human ECs impairs EC function, and inhibition of miR-92a can enhance vessel growth. One in vivo study with a mouse hind limb ischemia model revealed that the number of capillaries and smooth muscle actin-positive arterioles increases after antagomir-92a treatment. In searching for possible pathways and putative target genes for mir-92a, the mRNAs for integrin subunits a5 (ITGA5) and av, sphingosine-1phosphate receptor 1 (S1PR1), and mitogen-activated kinase 4 (MKK4) were found to show less expression in response to miR-92a overexpression. Further study on downstream pathways by silencing ITGA5 expression, resembling the effect of miR-92a on mRNA expression, revealed the expression levels of a second group of genes might be secondarily regulated as a consequence of ITGA5 downregulation. These genes include CD31, whose product is a platelet/endothelial cell adhesion molecule, and VWF, whose product is a Von Willebrand factor. The mir-92a-ITGA5-VWF axis could lead to low expression of Von Willebrand factor and cause instability of FVIII, finally resulting in prolonged APTT.

Lipocalin-2 has been studied as a clinical biomarker for DIC in its different forms: plasma free and total lipocalin2 levels were significantly higher in patients with overtDIC than in those without overt-DIC. The free lipocalin-2 level also showed a significant prognostic value in DIC [36]. In the search for a possible regulatory mechanism for mir-92a and lipocalin-2, studies have focused on apoptotic cell-derived sphingosine-1-phosphate (S1P), which induces the production and release of lipocalin-2 by human macrophages through the S1P-S1PR1-signal transducer and activator of transcription 3 (STAT3)-lipocalin-2 axis in lymphangiogenesis and tumor metastasis [37, 38]. Mir92a has been confirmed to play a role in the regulation of S1PR1 [35]. Increased mir-92a could lead to downregulation of S1PR1 and upregulation of lipocalin-2. However, this model still needs confirmation.

There are limitations to this study. First, without a basal level of miRNA expression in healthy controls, we could not determine whether the differently expressed miRNAs also differed between the SIC group and healthy controls in the same way. Second, because no optimum normalizer exists for such critical conditions as sepsis and severe infection [39], raw Ct value was taken into further analysis, which can cause bias due to individual difference and different sample degeneration states. 


\section{Conclusions}

In this work, we evaluated differently expressed miRNAs between SA and SIC patients. After validation, plasma hsa-mir-92a was confirmed as a diagnostic biomarker for sepsis-induced coagulopathy and related to global coagulation index and plasma lipocalin-2 level. Further study is needed to confirm the regulatory role of hsa-mir-92a-3p in coagulation disorders and assess therapeutic potency.

\section{Abbreviations}

APACHEIl: Acute Physiology and Chronic Health Evaluation; APTT: Activated Partial Thromboplastin Time; AUC: Area Under Curve; BGI: Beijing Genomics Institute; BUN: Blood Urea Nitrogen; Cl: Confidence Interval; DIC: Disseminated Intravascular Coagulation; ECs: Endothelial Cells; ELISA: Enzyme-Linked Immunosorbent Assay; FBG: Fibrinogen; ICU: Intensive Care Unit; INR: International Normalized Ratio; ITGA5: Integrin Subunits a5; miRNAs: MicroRNAs; MKK4: Mitogen-activated Kinase Kinase 4; NGAL: Neutrophil Gelatinase-Associated Lipocalin; PA: Prothrombin Activity; PT: Prothrombin Time; qRT-PCR: Quantitative Reverse TranscriptionPolymerase Chain Reaction; ROC: Receiver Operating Characteristic S1P: Sphingosine-1-Phosphate; S1PR1: Sphingosine-1-Phosphate Receptor 1; SA: Sepsis-Along; SIC: Sepsis-Induced Coagulopathy; SOFA: Sequential Organ Failure Assessment; STAT3: Signal Transducer and Activator of Transcription 3; TT: Thrombin Time; WWF: Von Willebrand Factor

\section{Acknowledgements}

Not applicable.

\section{Authors' contributions}

YW was in charge of study design, data analysis, and was a major contributor in writing the manuscript and experiment operation. HW helped with literature retrieval and revised the manuscript. CZ participated in design of the study and literature search. RG performed the literature search, data analysis and revised the manuscript. CFZ participated in conducting of Enzyme-linked immunosorbent assay. HY helped to revise the manuscript. ZT helped with study design and revised the manuscript. All authors have read and approved the final manuscript.

\section{Funding}

This work was supported by National Natural Science Foundation of China (NO. 81500003), Beijing Natural Science Foundation of China (NO. 7172084), the Beijing Municipal Administration of Hospitals Clinical Medicine Development of Special Funding Support (ID: ZYLX201312) and the Beijing Municipal Administration of Hospitals' Ascent Plan (ID: DFL20150302). The funders mentioned above financially sponsored this research in reagents, equipments, literature searching and also covered article processing fee.

\section{Availability of data and materials}

The datasets used and/or analysed during the current study are available from the corresponding author on reasonable request.

\section{Ethics approval and consent to participate}

The research related to human use has been complied with all the relevant national regulations, institutional policies and in accordance the tenets of the Helsinki Declaration, and has been approved by Ethics Committee of the Beijing Chaoyang Hospital. All of the patients gave their written informed consents.

\section{Consent for publication}

Not applicable.

\section{Competing interests}

The authors declare that they have no competing interests.

\section{Author details}

'Department of Respiratory and Critical Care Medicine, Beijing Engineering Research Center of Respiratory and Critical Care Medicine, Beijing Chao-Yang Hospital, Capital Medical University, Beijing Institute of Respiratory Medicine, NO. 8, Gong Ti South Road, Chao-Yang District, Beijing 100020, China.
${ }^{2}$ Department of Anesthesiology, Pain Medicine and Critical Care Medicine, Aviation General Hospital of China Medical University and Beijing Institute of Translational Medicine, Chinese Academy of Sciences, Beijing 100012, China.

Received: 12 October 2019 Accepted: 6 February 2020

Published online: 19 February 2020

\section{References}

1. Singer M, Deutschman CS, Seymour CW. The third international consensus definitions for Sepsis and septic shock (Sepsis-3). Jama. 2016;315(8):801-10.

2. Angus DC, van der Poll T. Severe sepsis and septic shock. N Engl J Med. 2013;369(9):840-51 2013

3. Dhainaut JF, Yan SB, Joyce DE. Treatment effects of drotrecogin alfa (activated) in patients with severe sepsis with or without overt disseminated intravascular coagulation. J Thromb Haemost. 2004;2(11):1924-33.

4. Yamakawa K, Umemura Y, Hayakawa M. Benefit profile of anticoagulant therapy in sepsis: a nationwide multicentre registry in Japan. Crit Care. 2016;20(1):229.

5. Iba T, Thachil J. Present and future of anticoagulant therapy using antithrombin and thrombomodulin for sepsis-associated disseminated intravascular coagulation: a perspective from Japan. Int J Hematol. 2016; 103(3):253-61.

6. Hayakawa M, Yamakawa K, Saito S. Recombinant human soluble thrombomodulin and mortality in sepsis-induced disseminated intravascular coagulation. A multicentre retrospective study. Thromb Haemost. 2016; 115(6):1157-66

7. Tagami T, Matsui $H$, Horiguchi $H$. Antithrombin and mortality in severe pneumonia patients with sepsis-associated disseminated intravascular coagulation: an observational nationwide study. J Thromb Haemost. 2014; 12(9):1470-9.

8. Iba T, Gando S, Thachil J. Anticoagulant therapy for sepsis-associated disseminated intravascular coagulation: the view from Japan. J Thromb Haemost. 2014;12(7):1010-9.

9. Nishida $\mathrm{O}$, Ogura $\mathrm{H}$, Egi $\mathrm{M}$. The Japanese clinical practice guidelines for Management of Sepsis and Septic Shock 2016. Acute Med Surg. 2018;5(1):3-89.

10. Lagos-Quintana M, Rauhut R, Lendeckel W. Identification of novel genes coding for small expressed RNAs. Science. 2001;294(5543):853-8.

11. Mitchell PS, Parkin RK, Kroh EM. Circulating microRNAs as stable bloodbased markers for cancer detection. Proc Natl Acad Sci U S A. 2008;105(30): 10513-8.

12. Chen $X, B a Y, M a L$. Characterization of microRNAs in serum: a novel class of biomarkers for diagnosis of cancer and other diseases. Cell Res. 2008;18(10): 997-1006.

13. Ma Y, Vilanova D, Atalar K. Genome-wide sequencing of cellular microRNAs identifies a combinatorial expression signature diagnostic of sepsis. PLoS One. 2013;8(10):e75918.

14. Wang HJ, Zhang PJ, Chen WJ. Evidence for serum miR-15a and miR-16 levels as biomarkers that distinguish sepsis from systemic inflammatory response syndrome in human subjects. Clin Chem Lab Med. 2012:50(8): 1423-8. https://doi.org/10.1515/cclm-2011-0826.

15. Wang HJ, Zhang PJ, Chen WJ. Four serum microRNAs identified as diagnostic biomarkers of sepsis. J Trauma Acute Care Surg. 2012;73(4):850-4.

16. Wang JF, Yu ML, Yu G. Serum miR-146a and miR-223 as potential new biomarkers for sepsis. Biochem Biophys Res Commun. 2010;394(1):184-8.

17. Wang L, Wang HC, Chen C. Differential expression of plasma miR-146a in sepsis patients compared with non-sepsis-SIRS patients. Exp Ther Med. 2013;5(4):1101-4.

18. Wang H, Meng K, Chen W. Serum miR-574-5p: a prognostic predictor of sepsis patients. Shock. 2012;37(3):263-7.

19. Kjeldsen $\mathrm{L}$, Johnsen $\mathrm{AH}$, Sengelov $\mathrm{H}$. Isolation and primary structure of lipocalin-2, a novel protein associated with human neutrophil gelatinase. $J$ Biol Chem. 1993;268(14):10425-32.

20. Lu F, Inoue K, Kato J. Functions and regulation of lipocalin-2 in gut-origin sepsis: a narrative review. Crit Care. 2019;23(1):269.

21. Chang W, Zhu S, Pan C. Predictive utilities of neutrophil gelatinaseassociated lipocalin (NGAL) in severe sepsis. Clin Chim Acta. 2018;481:200-6.

22. Iba T, Nisio M, Levy J. New criteria for sepsis-induced coagulopathy (SIC) following the revised sepsis definition: a retrospective analysis of a nationwide survey. BMJ Open. 2017:7(9):e017046.

23. Umemura Y, Yamakawa K, Ogura H. Efficacy and safety of anticoagulant therapy in three specific populations with sepsis: a meta-analysis of randomized controlled trials. J Thromb Haemost. 2016;14(3):518-30. 
24. Ding $R$, Wang $Z$, Lin $Y$. Comparison of a new criteria for sepsis-induced coagulopathy and international society on thrombosis and Haemostasis disseminated intravascular coagulation score in critically ill patients with sepsis 3.0: a retrospective study. Blood Coagul Fibrinolysis. 2018;29(6):551-8.

25. Liu R, Zhang C, Hu Z. A five-microRNA signature identified from genomewide serum microRNA expression profiling serves as a fingerprint for gastric cancer diagnosis. Eur J Cancer. 2011;47(5):784-91.

26. Zhu E, Zhao F, Xu G. MirTools: microRNA profiling and discovery based on high-throughput sequencing. Nucleic Acids Res. 2010;38:W392-7.

27. Zhang $\mathrm{G}$, Zhou $\mathrm{H}$, Xiao H. MicroRNA-92a functions as an oncogene in colorectal cancer by targeting PTEN. Dig Dis Sci. 2014;59(1):98-107.

28. Ohyashiki K, Umezu T, Yoshizawa S. Clinical impact of down-regulated plasma miR-92a levels in non-Hodgkin's lymphoma. PLoS One. 2011;6(2):e16408.

29. Yoshizawa S, Ohyashiki JH, Ohyashiki M. Downregulated plasma miR-92a levels have clinical impact on multiple myeloma and related disorders. Blood cancer. 2012;2(1):e53.

30. Nilsson S, Moller C, Jirstrom K. Downregulation of miR-92a is associated with aggressive breast cancer features and increased tumour macrophage infiltration. PLoS One. 2012;7(4):e36051.

31. Lin HY, Chiang CH, Hung WC. STAT3 upregulates miR-92a to inhibit RECK expression and to promote invasiveness of lung cancer cells. $\mathrm{Br} J$ Cancer. 2013;109(3):731-8

32. Hayashita $Y$, Osada $H$, Tatematsu Y. A polycistronic microRNA cluster, miR17-92, is overexpressed in human lung cancers and enhances cell proliferation. Cancer Res. 2005;65(21):9628-32.

33. Shigoka M, Tsuchida A, Matsudo T. Deregulation of miR-92a expression is implicated in hepatocellular carcinoma development. Pathol Int. 2010;60(5):351-7.

34. Chen ZL, Zhao XH, Wang JW. MicroRNA-92a promotes lymph node metastasis of human esophageal squamous cell carcinoma via E-cadherin. J Biol Chem. 2011;286(12):10725-34.

35. Bonauer A, Carmona G, Iwasaki M. MicroRNA-92a controls angiogenesis and functional recovery of ischemic tissues in mice. Science. 2009;324(5935):1710-3.

36. In JW, Kim JE, Jeong JS. Diagnostic and prognostic significance of neutrophil gelatinase-associated lipocalin-2 in disseminated intravascular coagulation. Clin Chim Acta. 2014;430:145-9.

37. Syed SN, Jung M, Weigert A. S1P provokes tumor Lymphangiogenesis via macrophage-derived mediators such as IL-1beta or Lipocalin-2. Mediat Inflamm. 2017;2017:7510496.

38. Jung M, Oren B, Mora J. Lipocalin-2 from macrophages stimulated by tumor cell-derived sphingosine 1-phosphate promotes lymphangiogenesis and tumor metastasis. Sci Signal. 2016;9(434):ra64.

39. Benz F, Roderburg C, Vargas CD. U6 is unsuitable for normalization of serum miRNA levels in patients with sepsis or liver fibrosis. Exp Mol Med. 2013;45:e42.

\section{Publisher's Note}

Springer Nature remains neutral with regard to jurisdictional claims in published maps and institutional affiliations.

Ready to submit your research? Choose BMC and benefit from:

- fast, convenient online submission

- thorough peer review by experienced researchers in your field

- rapid publication on acceptance

- support for research data, including large and complex data types

- gold Open Access which fosters wider collaboration and increased citations

- maximum visibility for your research: over $100 \mathrm{M}$ website views per year

At $\mathrm{BMC}$, research is always in progress.

Learn more biomedcentral.com/submissions 